\title{
Treatment of intraoperatively detected peritoneal carcinomatosis of colorectal origin with cytoreductive surgery and intraperitoneal chemotherapy
}

Aras Emre Canda ${ }^{{ }^{*}}$ (D), Cigdem Arslan ${ }^{1}$, Cem Terzi ${ }^{1}$, Selman Sokmen ${ }^{1}$, Tugba Yavuzsen², Sevda Ozkardesler ${ }^{3}$, Mehtat Unlư ${ }^{4}$ Funda Obuz ${ }^{5}$ and Mehmet Fuzun ${ }^{1}$

\begin{abstract}
Background: Diagnosis of peritoneal carcinomatosis (PC) may be missed by preoperative imaging. We are presenting our experience with incidentally detected PC of colorectal origin treated with cytoreductive surgery (CRS) and intraperitoneal chemotherapy (IPC) at the same operation.

Methods: Between January 2010 and September 2016, 19 patients underwent CRS and IPC due to incidentally detected PC of colorectal origin. Data were analyzed from a prospectively collected database.

Results: The median age was 59 (29-78). In three patients, PC was diagnosed during emergency surgery. The primary tumor was located in the rectum (three patients; one with recurrent disease), left colon (9 patients), and right colon (7 patients). All patients underwent CRS and IPC, and one patient operated laparoscopically. Median peritoneal cancer index (PCI) was 5 (range, 3-14), and complete cytoreduction (CC-0) was achieved in 14 patients. After CRS, 8 patients received early postoperative intraperitoneal chemotherapy (EPIC), 7 patients received hyperthermic intraperitoneal chemotherapy (HIPEC), and 4 patients received both HIPEC and EPIC. The median hospital stay was 9 (6-29) days. Postoperative complications occurred in 6 patients. There was no postoperative mortality. Median follow-up was 40.2 (12-94) months. Five-year overall survival was 63.2\%. Estimated mean survival time is longer in patients who underwent complete cytoreduction compared to patients having CC-1 or CC-2 cytoreduction (87.7 vs. 20.3 months; $p<0.001$ ).
\end{abstract}

Conclusion: Cytoreductive surgery and IPC can be performed safely in patients with intraoperatively detected incidental PC of colorectal origin.

Keywords: Peritoneal carcinomatosis, Intraperitoneal chemotherapy, HIPEC, EPIC, Colorectal, Incidental, Intraoperative detected

\section{Background}

Peritoneal carcinomatosis (PC) from colorectal cancer (CRC) has a poor prognosis and often considered as a terminal condition. Overall survival with current systemic chemotherapy regimens with new chemotherapeutic and molecular targeting agents varies between 13 and 34 months [1-3]. Currently, long-term survival can only

\footnotetext{
* Correspondence: emre.canda@deu.edu.tr

${ }^{1}$ Department of Surgery, Dokuz Eylul University School of Medicine, Balcova, 35340 Izmir, Turkey

Full list of author information is available at the end of the article
}

be achieved by cytoreductive surgery (CRS) and intraperitoneal chemotherapy (IPC). The incidence of synchronous PC in patients with colorectal cancer is 7\% [4]. Despite the advancements in imaging technics, the diagnostic accuracy of radiology in the identification of PC is still unsatisfying especially in patients having lowvolume disease. There are no recommendations in the guidelines or consensus reports for the management of the patients with unexpected peritoneal metastasis during surgery for CRC. In this study, we present our results of CRS and IPC in patients who underwent 
surgery for CRC with no preoperative suspicion of peritoneal metastasis.

\section{Methods \\ Patients' characteristics}

Between January 2010 and September 2016, we incidentally detected PC of colorectal origin in 24 patients during intraoperative exploration and performed CRS and IPC. We excluded patients with unresectable disease. Although all of the patients had preoperative computed tomography (CT) scans, we could not identify PC preoperatively. Peritoneal metastasis was confirmed in all patients, during working hours by frozen section and out of working hours by histopathology. In our department, we can perform CRS and IPC, and we routinely inform the patients for the need of multivisceral resections or CRS whom undergoing oncological surgery. Our main criteria for considering a patient unsuitable for CRS and IPC are the presence of diffuse small bowel or periportal involvement, unable to perform CC-0 and CC-1 cytoreduction, and extensive distant metastasis. The patients' performance status evaluated individually.

\section{Cytoreductive surgery}

The objective during CRS is to remove of all macroscopically visible tumor nodules from the visceral and parietal peritoneum by resection of the effected organ/ tissues or with peritonectomy procedures as previously described by Sugarbaker [5]. Electrosurgery was used for implants on visceral or intestinal surfaces where resection or excisions of the nodules were not possible. The extent of the peritoneal involvement was measured by peritoneal cancer index (PCI) [6]. After the completion of the resections and peritonectomy procedures, "Completeness of Cytoreduction" (CC) was classified as $\mathrm{CC}-0$, no residual disease; CC-1, minimal residual disease of $0-2.5 \mathrm{~mm}$; CC-2, residual disease of $2.5 \mathrm{~mm}-$ $2.5 \mathrm{~cm}$; and CC-3, residual disease $>2.5 \mathrm{~cm} \mathrm{[7].}$

\section{Intraperitoneal chemotherapy}

The rationale for performing IPC is to extend macroscopic disease elimination achieved by CRS to microscopic disease elimination. After the completion of cytoreduction, we delivered hyperthermic intraperitoneal chemotherapy (HIPEC) under general anesthesia with closed abdominal technique. Two inflow (one in the deep pelvis, one in the subhepatic or mostly affected area) and two outflow drains (one in the superficial pelvis site cavity) and two thermal probes were positioned in the abdominal cavity. The abdominal wall or skin was closed, although, after completion of the HIPEC, the surgical team would be able to re-explore or create gastrointestinal continuity at this site, if necessary. The drains and thermal probes were connected to the extracorporeal circuit of the HIPEC machine (Performer LRT, Rand, Italy). Three to five liters of perfusate were used depending on the abdominal cavity volume. Our oncologist coordinated chemotherapeutic dose for every individual patient. We used oxaliplatin at a dose of $430 \mathrm{mg} / \mathrm{m}^{2}$ at $42-43{ }^{\circ} \mathrm{C}$ intracavitary temperature for $30 \mathrm{~min}$ for peritoneal metastases from the colorectal origin. For early postoperative intraperitoneal chemotherapy (EPIC), we placed four outflow drains at the same position as in HIPEC and a Jackson-Pratt drain subhepatic space as an inflow catheter. In the surgical ward, a peritoneal infusion in 11 of $0.09 \mathrm{NaCl}$ was given at day 0 from the Jackson-Pratt drain in order to prevent intraperitoneal adhesions. Then, 5-FU $\left(650 \mathrm{mg} / \mathrm{m}^{2}\right)$ and sodium bicarbonate in 11 of $0.09 \mathrm{NaCl}$ were given intraperitoneally in the next 5 days. These drugs remained in place for $23 \mathrm{~h}$ before drainage for $1 \mathrm{~h}$ before the next infusion.

\section{Evaluation of complications and toxicity}

According to our protocol for CRS and IPC, we record complications, systemic toxicities, and mortality occurring during the postoperative hospital stay or within 30 days of operation. We retrospectively analyzed those data.

\section{Oncological follow-up}

Follow-up included a physical examination and CEA measurements every 3 months for the first year, twice a year afterward. A CT scan of the abdomen and thorax every 6 months for the first 2 years and yearly after that. We perform colonoscopy at the end of year 1. Magnetic resonance imaging or positron emission $\mathrm{CT}$ is not routine imaging tools and performed when necessary. The exact status of each patient was retrospectively analyzed from a specific database of Surgery and Oncology Departments.

\section{Statistical analysis}

Continuous variables were expressed as means and minimum and maximum values (range) and categorical variables as frequency and percentages. Patients' data were compiled into a computer statistical software including demographic, surgical, pathologic, and survival figures. Survival rates were calculated using KaplanMeier method and were compared with the log-rank test $(p<0.05$ was considered statistically significant).

\section{Results}

Nineteen patients were included in the analysis. Flow diagram of the study is given in Fig. 1. The median age of the patients was 59 (range; 29-78) years, and five of them were female. Five patients had comorbidities including chronic obstructive pulmonary disease (one patient), diabetes mellitus (two patients), and hypertension (three patients). Median American Society of Anesthesiologists (ASA) score was 1 (range, 1-3). The primary tumor was 


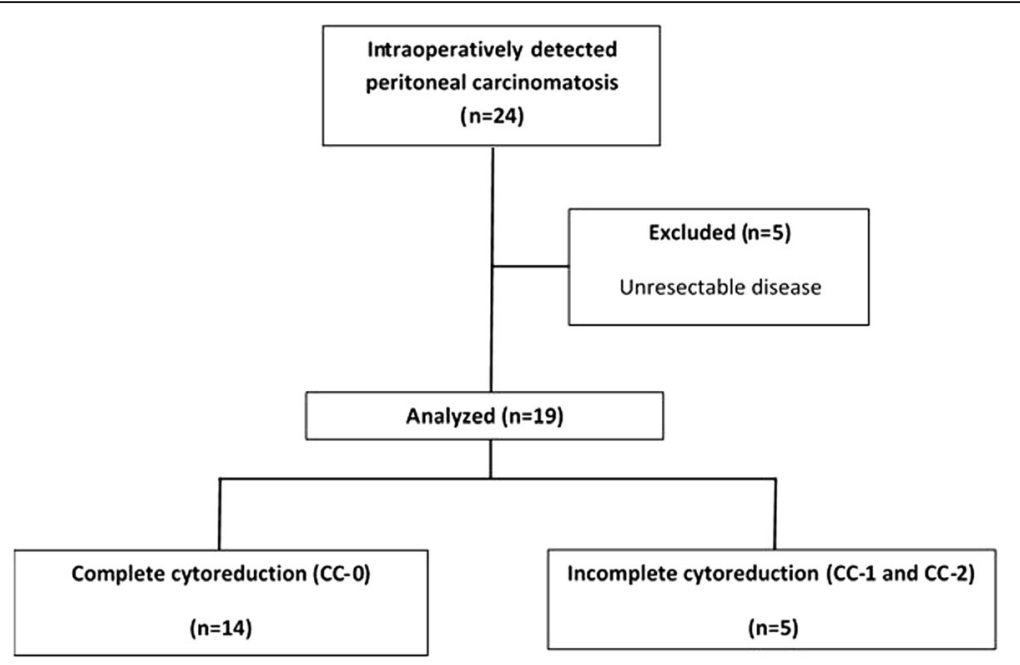

Fig. 1 Flow diagram of the study

located in the rectum (three patients), left colon (9 patients), and right colon (7 patients). Two patients with locally advanced rectal cancer received preoperative chemoradiotherapy. One patient underwent surgery for recurrent rectal cancer; others had a primary disease. In three patients, $\mathrm{PC}$ was diagnosed during emergency surgery. All patients underwent CRS and IPC, and one patient operated laparoscopically. We performed Hartmann procedure in 2 patients and diverting loop ileostomy in one patient which was closed to 6 following the initial procedure. Median PCI was 5 (range, 3-14). We achieved macroscopically complete cytoreduction (CC-0) in 14 patients. After completion of CRS, we performed IPC, EPIC in 8 patients, HIPEC in 7 patients, and both HIPEC and EPIC in four patients. None of the patients stayed in intensive care unit; the median hospital stay was 9 days (range, 6-29) (Table 1).

Postoperative complications occurred in 6 patients. These included surgical site infection in four patients, urinary tract infection in two patients, chylous drainage in one patient, and small intestine perforation in one patient (she underwent a reoperation). No WHO grade 3 or 4 bone marrow or renal toxicities were observed. We did not find any difference related to different types of IPC regarding postoperative complications. No patients died during the perioperative period.

Based on the histological examination, primary tumor stage was $\mathrm{T} 3$ in three patients and T4 in 16 patients and 10 patients had lymph node metastases. Postoperatively, all patients received further systemic chemotherapy.

Median follow-up time was 40.2 (range, 3-94) months. Two patients developed isolated local recurrence, four patients developed isolated distant metastasis, and two patients developed combined local recurrence and distant metastasis. Median time to local recurrence and distance metastasis was 5 (3-14) and 11 (8-24) months. Two patients with $\mathrm{CC}-1$ and $\mathrm{CC}-2$ resections died due to progressive disease; three patients, due to locally recurrent; and two patients, due to metastatic disease. Two patients were alive with persistent disease, and 10 patients were alive without any evidence of disease. Estimated mean $( \pm \mathrm{SE})$ survival time was $64.5( \pm 8.4)$ months

Table 1 Demographic and surgical characteristics of the patients

\begin{tabular}{|c|c|}
\hline Variables & \\
\hline \multicolumn{2}{|l|}{ Sex } \\
\hline Male & 14 \\
\hline Female & 5 \\
\hline Age (year) & $59(29-78)$ \\
\hline ASA score & $1(1-3)$ \\
\hline \multicolumn{2}{|l|}{ Tumor origin } \\
\hline Right colon & 7 \\
\hline Left colon & 9 \\
\hline Rectum & 3 \\
\hline Median PCl score & $5(3-14)$ \\
\hline \multicolumn{2}{|l|}{ Completeness of cytoreduction } \\
\hline CC-O & 14 \\
\hline CC-1 & 4 \\
\hline CC-2 & 1 \\
\hline \multicolumn{2}{|c|}{ Type of intraperitoneal chemotherapy } \\
\hline EPIC & 8 \\
\hline HIPEC & 7 \\
\hline EPIC + HIPEC & 1 \\
\hline Median hospital stay (days) & $9(6-29)$ \\
\hline
\end{tabular}

ASA American Society of Anesthesiologists, $\mathrm{PCl}$ peritoneal carcinomatosis index, EPIC early postoperative intraperitoneal chemotherapy, HIPEC hyperthermic intraperitoneal chemotherapy 
with a 5-year survival rate of $63.2 \%$ (Fig. 2). Estimated mean survival time is longer in patients who had no lymph node metastasis compared to patients having lymph node metastasis (77.1 vs. 37.8 months); however, the difference did not reach statistical significance $(p=0$. 428). Estimated mean survival time is significantly longer in patients who had complete cytoreduction compared to patients having $\mathrm{CC}-1$ or $\mathrm{CC}-2$ cytoreduction (78 vs. 20 months; $p<0.001$ ) (Fig. 3).

\section{Discussion}

The peritoneum is the second most common site after the liver of colorectal cancer metastases [8]. The natural history of the disease has a poor median survival of approximately 6 months which is increased up 34 months with new systemic chemotherapy regimens $[1-3,9,10]$. However, long-term survival is still hard to be achieved by systemic chemotherapy alone. Elias et al. reported $60 \%$ 2-year survival with cytoreductive surgery with or without EPIC in patients with CRC and PC [11]. Longterm follow-up results of a randomized controlled trial showed $45 \%$ disease-free survival rates in CRS and HIPEC arm compared to less than $10 \%$ in incomplete cytoreduction or systemic chemotherapy arm [12]. A recent meta-analysis confirmed the improvement of survival with CRS and HIPEC in selected patients with peritoneal carcinomatosis from colorectal cancer [13]. Peritoneal Surface Oncology Group International (PSOGI) reached a consensus that CRS and HIPEC should be considered as the standard therapy for the selected patients with mild-to-moderate peritoneal metastasis [14]. The addition of EPIC to HIPEC may provide an increase in survival but increases the morbidity [15].

Currently, standard preoperative radiologic tool for staging colorectal cancer is CT [16]. The sensitivity of CT for detecting PC is $60-90 \%$ and influenced by the extent of the disease, size, and site of the nodules [17, 18]. Although multi-detector CT enables more accurate images, the extent of the PC is underestimated in approximately one third of the patients [18-20]. The accuracy of CT

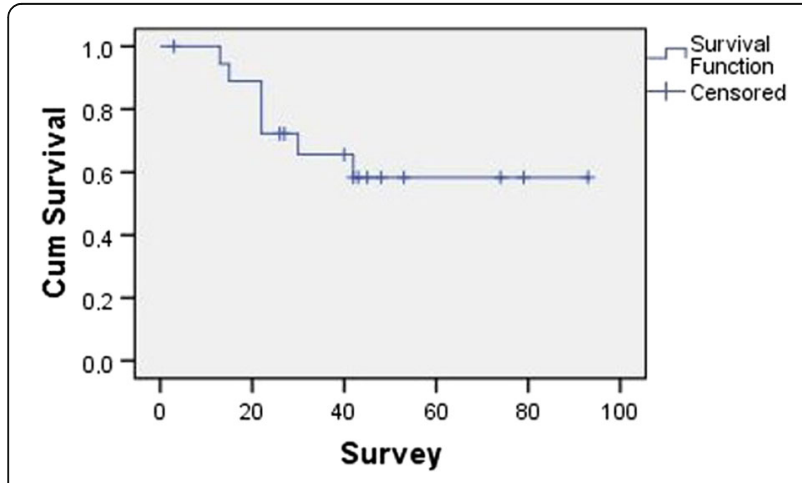

Fig. 2 Kaplan-Meier survival curve for median survival decreases by the size of the implants, particularly in right-upper quadrant, right-lower quadrant, left-lower quadrant, distal jejunum, and distal ileum [18]. Tumor nodules $<5 \mathrm{~mm}$ and small-bowel mesentery location have detection sensitivities as low as $10 \%$ with CT $[19,20]$. Despite the advancements in imaging technics, we still face with unexpected PC intraoperatively.

There are no clear recommendations or publications for the management of the patient with intraoperatively detected and unexpected peritoneal metastasis of CRC. Closing the abdomen with only a biopsy and reference to a tertiary center having access to HIPEC or preoperative systemic chemotherapy are alternatives. Initial surgery should be as sparing as possible, in order not to damage the peritoneal surface and to evoke intraperitoneal release of growth factors [21]. Unnecessary dissection and resections may result with adhesions which may be a challenge for the surgeon who will going to perform CRS. On the other hand, considering the low tumor burden which could not be detected by CT, most of these patients would be suitable candidates for CRS and IPC. In the presence of experienced surgical team and sufficient technical settings including $7 / 24$ available oncology consultant and chemotherapeutical agents, intraoperatively detected PC can be treated by CRS and IPC at once at the same surgery. In our center, we can offer CRS and IPC for those patients. Several factors influence the choice of IPC. When we detect PC incidentally and if we operate the patient in working hours, we are able to perform a frozen section to confirm the peritoneal metastases and consult the patient intraoperatively with the oncologist, and we can deliver HIPEC. If we detect PC out of the working hours, we are not able to perform a frozen section so we get biopsies from the implants and deliver EPIC after the histopathological confirmation at the following days. Also, reimbursement of HIPEC is a problem in our country and conditions vary, so we perform HIPEC when the patient's insurance or the patient individually pays. We prefer the addition of EPIC to HIPEC in patients with incomplete cytoreduction.

Postoperative morbidity and mortality have been reported $12-56$ and $0-12 \%$ in the literature [22]. Overall morbidity and mortality were reported 39.0-48.5 and $6.5-7.6 \%$ in previously published studies of our group $[23,24]$. In the present study, morbidity was lower than our entire CRS and IPC series, presumably due to lower PCI scores requiring less aggressive surgery and shorter operative times. There was no postoperative mortality.

We diagnosed synchronous PC during emergency surgery for the primary tumor in three patients. Due to low sample size, we did not perform a comparison in terms of operative outcomes, postoperative complications, toxicities, adjuvant therapies, and survival. Van Oudheusden et al. reported their results in patients who underwent 


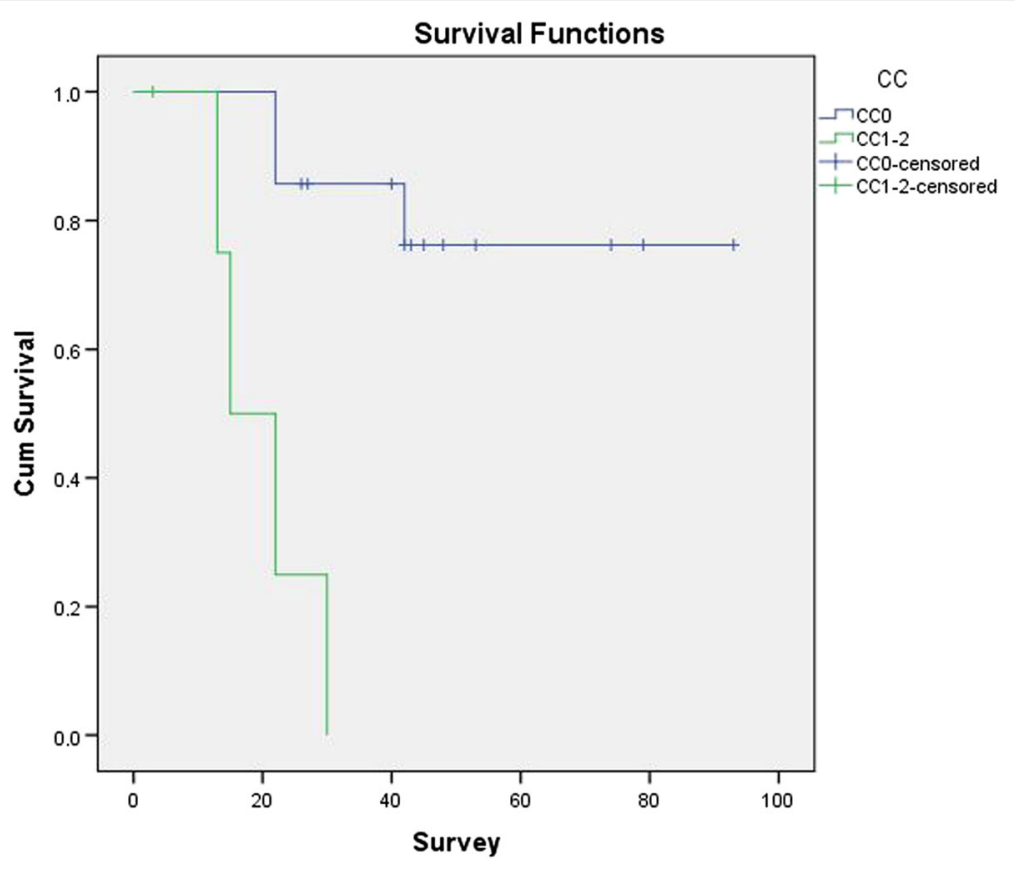

Fig. 3 Kaplan-Meier survival curves following complete and incomplete cytoreduction

CRS and HIPEC after emergency surgery in the presence of PC, and they observed similar operative outcomes, postoperative complications, and survival compared with the patients in whom PC was diagnosed in an elective setting [21]. When performed by a specialized team, CRS and IPC are a safe procedure in selected patients with $\mathrm{PC}$ from colorectal origin.

The PCI score and completeness of cytoreduction have been shown to be associated with better survival in several studies $[25,26]$. The expected extent of the disease in patients with incidentally found PC is low, insomuch preoperatively undetectable. Therefore, those patients have the best potential to have a curative treatment of PC. In our study, the median PCI was 5, and unsurprisingly, estimated 5-year overall was favorable when compared with the literature $[11,13]$. We found significantly longer mean survival in patients who underwent complete cytoreduction compared to the patients having CC-1 or CC-2 cytoreduction.

\section{Conclusion}

There is a demand for management of intraoperatively detected PC. Cytoreductive surgery and IPC can be performed safely in patients with intraoperatively detected incidental PC of colorectal origin. A multidisciplinary team work, on-demand availability of frozen section, intraoperative oncology consultation, and HIPEC machine are essential for intraoperative management of PC. Controlled trials are needed to identify the best timing of definitive treatment.
Abbreviations

ASA: American Society of Anesthesiologists; CC: Completeness of Cytoreduction; CRC: Colorectal cancer; CRS: Cytoreductive surgery; CT: Computed tomography; EPIC: Early postoperative intraperitoneal chemotherapy; HIPEC: Hyperthermic intraperitoneal chemotherapy; IPC: Intraperitoneal chemotherapy; PC: Peritoneal carcinomatosis; PCl: Peritoneal cancer index; PSOGI: Peritoneal Surface Oncology Group International

Acknowledgements

There are no acknowledgements for this study.

Funding

We do not have a funding for this study.

Availability of data and materials

We may share our data. Please contact the author for data requests.

\section{Authors' contributions}

AEC and CA designed and drafted the manuscript and performed the statistical analysis. CT, SSN, TY, SO, MU, and FO helped to draft the manuscript and made a critical review. All authors read and approved the final manuscript.

Ethics approval and consent to participate

This study is approved by the Dokuz Eylul University Non-interventional Studies Ethics Committee (2012/34-03).

Consent for publication

Not applicable

\section{Competing interests}

The authors declare that they have no competing interests.

\section{Publisher's Note}

Springer Nature remains neutral with regard to jurisdictional claims in published maps and institutional affiliations. 


\section{Author details}

'Department of Surgery, Dokuz Eylul University School of Medicine, Balcova, 35340 Izmir, Turkey. ${ }^{2}$ Department of Medical Oncology, Dokuz Eylul University School of Medicine, Balcova, 35340 Izmir, Turkey. ${ }^{3}$ Department of Anaesthesiology and Reanimation, Dokuz Eylul University School of Medicine, Balcova, 35340 Izmir, Turkey. ${ }^{4}$ Department of Pathology, Dokuz Eylul University School of Medicine, Balcova, 35340 Izmir, Turkey. 5epartment of Radiodiagnostics, Dokuz Eylul University School of Medicine, Balcova, 35340 Izmir, Turkey.

Received: 3 November 2017 Accepted: 16 March 2018 Published online: 27 March 2018

\section{References}

1. Franko J, Shi Q, Goldman CD, Pockai BA, Nelson GD, Goldberg RM, et al. Treatment of colorectal peritoneal carcinomatosis with systemic chemotherapy: a pooled analysis of North Central Cancer Treatment Group phase III trials N9741 and N9841. J Clin Oncol. 2012;30:263-7.

2. Schwartzberg LS, Rivera F, Karthaus M, Fasola G, Canon JL, Hecht JR, et al. PEAK: a randomized, multicenter phase II study of panitumumab plus modified fluorouracil, leucovorin, and oxaliplatin (mFOLFOX6) or bevacizumab plus mFOLFOX6 in patients with previously untreated, unresectable, wild-type KRAS exon 2 metastatic colorectal cancer. J Clin Oncol. 2014;32(21):2240-7.

3. Douillard JY, Siena S, Cassidy J, Tabernero J, Burkes R, Barugel M, et al. Final results from PRIME: randomized phase III study of panitumumab with FOLFOX4 for first-line treatment of metastatic colorectal cancer. Ann Oncol. 2014;25(7):1346-55.

4. Quere P, Facy O, Manfredi S, Jooste V, Faivre J, LEpage C, et al. Epidemiology, management, and survival of peritoneal carcinomatosis from colorectal cancer: a population-based study. Dis Colon Rectum. 2015;58:743-52.

5. Sugarbaker PH. Peritonectomy procedures. Ann Surg. 1995;221:29-42.

6. Jacquet $\mathrm{P}$, Sugarbaker $\mathrm{PH}$. Clinical research methodologies in diagnosis and staging of patients with peritoneal carcinomatosis. In: Sugarbaker PH, editor. Peritoneal carcinomatosis: principles of management. Boston: Kluwer Academic; 1996. p. 359-74

7. Jacquet P, Sugarbaker PH. Current methodologies for clinical assessment of patients with peritoneal carcinomatosis. J Exp Clin Cancer Res. 1996;15:49-58.

8. Thomassen I, van Gestel YR, Lemmens VE, de Hingh $\mathrm{H}$. Incidence, prognosis, and treatment options for patients with synchronous peritoneal carcinomatosis and liver metastases from colorectal origin. Dis Colon Rectum. 2013;56:1373-80.

9. Chu DZ, Lang NP, Thompson C, Osteen PK, Westbrook KC. Peritoneal carcinomatosis in nongynecologic malignancy. A prospective study of prognostic factors. Cancer. 1989;63:364-7.

10. Sadeghi B, Arvieux C, Glehen O, Beaujar AC, Rivoire M, Baulieux J, et al. Peritoneal carcinomatosis from non-gynecologic malignancies: results of the EVOCAPE 1 multicentric prospective study. Cancer. 2000;88:358-63.

11. Elias D, Lefevre JH, Chevalier J, Brouget A, Marchal F, et al. Complete cytoreductive surgery plus intraperitoneal chemohyperthermia with oxaliplatin for peritoneal carcinomatosis of colorectal origin. J Clin Oncol. 2009;27:681-5.

12. Verwaal VJ, Bruin S, Boot H, van Slooten G, van Tinteren H. 8-Year follow-up of randomized trial: cytoreduction and hyperthermic intraperitoneal chemotherapy versus systemic chemotherapy in patients with peritoneal carcinomatosis of colorectal cancer. Ann Surg Oncol. 2008;9:2426-32.

13. Huang CQ, Min Y, Wang SY, Yang XJ, Liu Y, Xiong B, et al. Cytoreductive surgery plus hyperthermic intraperitoneal chemotherapy improves survival for peritoneal carcinomatosis from colorectal cancer: a systematic review and meta-analysis of current evidence. Oncotarget. 2017;8:55657-83.

14. Li Y, Yu Y, Liu Y. Report on the 9(th) international congress on peritoneal surface malignancies. Cancer Biol Med. 2014;11:281-4.

15. Lam JY, McConnell YJ, Rivard JD, Temple WJ, Mack LA. Hyperthermic intraperitoneal chemotherapy + early postoperative intraperitoneal chemotherapy versus hyperthermic intraperitoneal chemotherapy alone: assessment of survival outcomes for colorectal and high-grade appendiceal peritoneal carcinomatosis. Am J Surg. 2015;210(3):424-30.

16. NCCN, "Rectal Cancer NCCN 2016 v2,", 2016
17. De Bree E, Koops W, Kröger R, Van Ruth S, Witkamp AJ, Zoetmulder FAN. Peritoneal carcinomatosis from colorectal or appendiceal origin: correlation of preoperative $C T$ with intraoperative findings and evaluation of interobserver agreement. J Surg Oncol. 2004;86:64-73.

18. Esquivel J, Chua TC, Stojadinovic A, Melero JT, Levine EA, Gutman M, et al. Accuracy and clinical relevance of computed tomography scan interpretation of peritoneal cancer index in colorectal cancer peritoneal carcinomatosis: a multi-institutional study. J Surg Oncol. 2010;6:565-70.

19. Marin D, Catalano C, Baski M. 64-Section multi-detector row CT in the preoperative diagnosis of peritoneal carcinomatosis: correlation with histopathological findings. Abdom Imaging. 2010;6:694-700.

20. Koh JL, Yan TD, Glenn D, Morris DL. Evaluation of preoperative computed tomography in estimating peritoneal cancer index in colorectal peritoneal carcinomatosis. Ann Surg Oncol. 2009;16:327-33.

21. van Oudheusden TR, Braam HJ, Nienhuiis SW, Wiezer MJ, van Ramshorst B, Luyer MD, et al. Cytoreduction and hyperthermic intraperitoneal chemotherapy: a feasible and effective option for colorectal cancer patients after emergency surgery in the presence of peritoneal carcinomatosis. Ann Surg Oncol. 2014;21:2621-6.

22. Chua TC, Yan TD, Saxena A, Morris DL. Should the treatment of peritoneal carcinomatosis by cytoreductive surgery and hyperthermic intraperitoneal chemotherapy still be regarded as a highly morbid procedure?: a systematic review of morbidity and mortality. Ann Surg. 2009;249:900-7.

23. Canda AE, Sokmen S, Terzi C, Arslan C, Oztop I, Karabulut B. Complications and toxicities after cytoreductive surgery and hyperthermic intraperitoneal chemotherapy. Ann Surg Oncol. 2013;20:1082-7.

24. Arslan NC, Sokmen S, Avkan-Oguz V, Obuz F, Canda AE, Terzi C, et al. Infectious complications after cytoreductive surgery and hyperthermic intra-peritoneal chemotherapy. Surg Infect. 2017;18:157-63.

25. Frøysnes IS, Larsen SG, Spasojevic M, Dueland S, Flatmark K. Complete cytoreductive surgery and hyperthermic intraperitoneal chemotherapy for colorectal peritoneal metastasis in Norway: prognostic factors and oncologic outcome in a national patient cohort. J Surg Oncol. 2016;114:222-7.

26. Harmon RL, Sugarbaker PH. Prognostic indicators in peritoneal carcinomatosis from gastrointestinal cancer. Int Semin Surg Oncol. 2005;2:3.

\section{Submit your next manuscript to BioMed Central and we will help you at every step:}

- We accept pre-submission inquiries

- Our selector tool helps you to find the most relevant journal

- We provide round the clock customer support

- Convenient online submission

- Thorough peer review

- Inclusion in PubMed and all major indexing services

- Maximum visibility for your research

Submit your manuscript at www.biomedcentral.com/submit
) Biomed Central 